\title{
On foliations in Sikorski differential spaces with Brouwerian leaves
}

\author{
by WŁodzimierz WALISzEWSKI (Łódź)
}

\begin{abstract}
The class of locally connected and locally homeomorphically homogeneous topological spaces such that every one-to-one continuous mapping of an open subspace into the space is open has been considered. For a foliation $F$ [3] on a Sikorski differential space $M$ with leaves having the above properties it is proved that for some open sets $U$ in $M$ covering the set of all points of $M$ the connected components of $U \cap \underline{L}$ in the topology of $M$ coincide with the connected components in the topology of $L$ for $L \in F$.
\end{abstract}

1. Brouwerian topological spaces. For a topological space $X$ the set of all points of $X$ will be denoted by $\underline{X}$. A continuous mapping $f: X \rightarrow Y$ is said to be open iff for any open set $A$ in $X$ the set $f(A)$ is open in $Y$. A topological space $X$ is said to be locally homeomorphically homogeneous (l.h.h.) iff for any $p, q \in \underline{X}$ there exists a homeomorphism $h: U \rightarrow V$ such that $p \in U, q \in V, h(p)=q, U$ and $V$ are open subspaces of $X$. A set $\mathcal{T}$ of topological spaces will be called l.h.h. iff the disjoint union $\bigoplus \mathcal{T}$ of $\mathcal{T}$ is l.h.h. A locally connected l.h.h. topological space $X$ such that every continuous 1-1 mapping $f: V \rightarrow X$ of an open subspace $V$ of $X$ into $X$ is open will be called Brouwerian.

A set $\mathcal{T}$ of topological spaces such that the disjoint union $\bigoplus \mathcal{T}$ is Brouwerian will be called Brouwerian. By Brouwer's well-known theorem on open mappings in $\mathbb{R}^{n}$ every topological manifold is Brouwerian.

Example $1 . \underline{X}=\{0,1\}$. The topology of $X$ is of the form $\{\emptyset, \underline{X}\} . X$ is Brouwerian but not a topological manifold.

The topological space induced by $X$ in the set $A$ is denoted by $X \mid A$. The set of all connected components of $X$ will be denoted by $\operatorname{cc}(X)$.

By an easy verification we have

Proposition 1. If $X$ is l.h.h. and a non-empty open subspace of $X$ is Brouwerian then $X$ is Brouwerian.

1985 Mathematics Subject Classification: Primary 53C12. 
As an immediate corollary of Proposition 1 we get

Proposition 2. An l.h.h. set $\mathcal{T}$ of topological spaces is Brouwerian iff there exists a Brouwerian space belonging to $\mathcal{T}$.

Proposition 2 together with the remark that the disjoint union of a set of mutually homeomorphic Brouwerian spaces is Brouwerian allows us to construct a Brouwerian space with an arbitrary infinite cardinal number of the set of points being not a topological manifold. Moreover, we construct a Sikorski differential structure [1] with the topology having the above features.

ExAmple 2. Let $I$ be any set and let $I^{\prime}$ be the set of all real functions $\alpha$ defined on $\{0,1\} \times I$ and such that $\alpha(0, i)=\alpha(1, i)$ for $i \in I$.

It is easy to check that the set $I^{\prime}$ is a Sikorski differential structure on $\{0,1\} \times I$. The topology of this structure, i.e. the weakest topology for which all the functions of $I^{\prime}$ are continuous, is the topology of $\bigoplus_{i \in I} I_{i}$, where $I_{i}$ is the topological space with $\{0,1\} \times\{i\}$ as the set of all points and the topology $\{\emptyset,\{0,1\} \times\{i\}\}$, i.e. $I_{i}$ is homeomorphic to the space in Example 1.

Proposition 3. If $\mathcal{T}$ is a Brouwerian set of topological spaces such that $\underline{X} \cap \underline{X}^{\prime}=\emptyset$ when $X \neq X^{\prime}, X, X^{\prime} \in \mathcal{T}, T$ is a topological space satisfying

$$
\underline{T}=\bigcup_{X \in \mathcal{T}} \underline{X}
$$

$$
\operatorname{id}_{\underline{X}}: X \rightarrow T \quad \text { for } X \in \mathcal{T},
$$

and there exists a homeomorphism

$$
g: T \rightarrow Y \times S
$$

where $Y$ is Brouwerian, $S$ is a topological space and

$$
\bigcup_{X \in \mathcal{T}} \operatorname{cc}(T \mid \underline{X})=\left\{g^{-1}(\underline{Y} \times\{s\}) ; s \in \underline{S}\right\},
$$

then

$$
\bigcup_{X \in \mathcal{T}} \operatorname{cc}(T \mid \underline{X})=\bigcup_{X \in \mathcal{T}} \operatorname{cc}(X) .
$$

Proof. Let $A \in \operatorname{cc}(X), X \in \mathcal{T}$. Because of the local connectedness of $X$ we see that $A$ is open in $X$ and $X \mid A$ is connected.

So, by (2), $T \mid A$ is connected. Let $A \subset \widetilde{A} \in \operatorname{cc}(T \mid \underline{X})$. By (4) there is exactly one $w_{A} \in \underline{S}$ such that $\widetilde{A}=g^{-1}\left(\underline{Y} \times\left\{w_{A}\right\}\right)$. Therefore,

$$
A \subset g^{-1}\left(\underline{Y} \times\left\{w_{A}\right\}\right) .
$$


Take any $C \in \bigcup_{X \in \mathcal{T}} \operatorname{cc}(T \mid \underline{X})$. Setting

$$
\widehat{C}=\left\{A ; A \in \bigcup_{X \in \mathcal{T}} \operatorname{cc}(X) \text { and } w_{A}=s\right\},
$$

where

$$
C=g^{-1}(\underline{Y} \times\{s\}), s \in \underline{S},
$$

by (6)-(8) we get $A \subset C$ for $A \in \widehat{C}$. Then $\bigcup \widehat{C} \subset C$. On the other hand, taking any $c \in C$, by (1) we get $X \in \mathcal{T}$ with $c \in \underline{X}$. Thus there exists $A \in \operatorname{cc}(X)$ with $c \in A$. According to (6), $c \in g^{-1}\left(\underline{Y} \times\left\{w_{A}\right\}\right)$. Hence, by (8), $w_{A}=s$. Therefore, $c \in A \in \widehat{C}$. Thus, $C \subset \cup \widehat{C}$. Hence,

$$
C=\bigcup \widehat{C} \text {. }
$$

From local connectedness of all topological spaces belonging to $\mathcal{T}$, by $(7)$ and (9) it follows that $C$ is open in $X$. The homeomorphism (3) induces the following one:

$$
g|C: T| C \rightarrow Y \times S \mid\{s\} .
$$

Taking continuous 1-1 mappings $\operatorname{id}_{C}: X|C \rightarrow T| C$ and $\operatorname{pr}_{1}: Y \times S \mid\{s\} \rightarrow Y$ we get

$$
\operatorname{pr}_{1} \circ g\left|C \circ \operatorname{id}_{C}: X\right| C \rightarrow Y .
$$

From Proposition 2 we find that the mapping (10) is open. Therefore (10) is a homeomorphism. Thus $X \mid C$ is connected. To prove that $C \in \operatorname{cc}(X)$ take any $H$ connected in $X$ with $C \subset H$. Then, by (2), $H$ is connected in $T$. Therefore there is $C_{0} \in \operatorname{cc}(T \mid \underline{X})$ with $H \subset C_{0}$. By (4), we get $C_{0}=g^{-1}\left(\underline{Y} \times\left\{s_{0}\right\}\right), s_{0} \in S$. From $\emptyset \neq C \subset C_{0}$ and (8) it follows that $s=s_{0}$. Thus $C_{0}=C$. This yields $H \subset C$. Therefore $C \in \operatorname{cc}(X)$. Thus,

$$
\bigcup_{X \in \mathcal{T}} \operatorname{cc}(T \mid \underline{X}) \subset \bigcup_{X \in \mathcal{T}} \operatorname{cc}(X) \text {. }
$$

The families of sets on the left as well as on the right of the inclusion (11) are partitions of the same set $\underline{T}$. Hence it follows that the inverse inclusions is true.

2. Connected components in distinguished sets of a foliation. For a Sikorski differential space (d.s.) $M$ the set of all points of $M$ and the differential structure of $M$ are denoted by $\underline{M}$ and $F(M)$, respectively. For any set $A \subset \underline{M}$ the d.s. induced by $M$ on $A$, i.e. the d.s. $\left(A, F(M)_{A}\right)$, is denoted by $\overline{M_{A}}$. We recall the concept of foliation in the category of d.s. [3].

Let $M$ be a d.s. and let $\mathcal{F}$ be a set of disjoint d.s. such that $\underline{M}=\bigcup_{L \in \mathcal{F}} \underline{L}$. $\mathcal{F}$ is assumed to be locally homogeneous (1.h.), i.e. for any $K, L \in \mathcal{F}, p \in \underline{K}$ and $q \in \underline{L}$ there exists a diffeomorphism $h: K_{A} \rightarrow L_{B}$, where $p \in A \in$ top $K$, 
$q \in B \in \operatorname{top} L$ and $h(p)=q$. A set $U \in$ top $M$ will be called distinguished by $\mathcal{F}$ iff there exist $K \in \mathcal{F}, V \in \operatorname{top} K$, a d.s. $N$ and a diffeomorphism

$$
\Phi: M_{U} \rightarrow K_{V} \times N
$$

such that

$$
\bigcup_{L \in \mathcal{F}} \operatorname{cc}(\operatorname{top} M \mid U \cap \underline{L})=\left\{\Phi^{-1}(V \times\{b\}) ; b \in \underline{N}\right\} .
$$

The set $\mathcal{F}$ is said to be a foliation on $M$ iff

(i) $L$ is connected and regularly lying [2] in $M$ for $L \in \mathcal{F}$, i.e. $\operatorname{id}_{\underline{L}}: L \rightarrow$ $M$ is regular;

(ii) for any $p \in \underline{M}$ there exist $K \in \mathcal{F}, V \in$ top $K$ with $p \in V$ and a diffeomorphism (12) satisfying (13).

From (ii) it follows that $M$ is covered by open sets distinguished by $\mathcal{F}$.

Theorem. If $\mathcal{F}$ is a Brouwerian foliation on $M$ then for any open set $U$ in $M$ distinguished by $\mathcal{F}$ we have

$$
\bigcup_{L \in \mathcal{F}} \operatorname{cc}(\operatorname{top} M \mid U \cap \underline{L})=\bigcup_{L \in \mathcal{F}} \operatorname{cc}(\operatorname{top} L \mid U \cap \underline{L}) .
$$

Proof. For a set $U$ distinguished by $\mathcal{F}$ we have a diffeomorphism (12) with (13). Setting, in Proposition $3, T=\operatorname{top} M_{U}, Y=\operatorname{top} K_{V}, S=$ top $N, \mathcal{T}=\{\operatorname{top} L \mid U \cap \underline{L} ; L \in \mathcal{F}\}$ and the homeomorphism (3) as the one induced by the diffeomorphism (12) we get (4) and, consequently, (5).

Remark. In the proof of the Theorem the regularity of $\operatorname{id}_{\underline{L}}: L \rightarrow M$ for $L \in F$ has not been essential.

\section{References}

[1] R. Sikorski, Abstract covariant derivative, Colloq. Math. 18 (1967), 251-272.

[2] W. Waliszewski, Regular and coregular mappings of differential spaces, Ann. Polon. Math. 30 (1975), 263-281.

[3] —, Foliations of differential spaces, Demonstratio Math. 18 (1) (1985), 347-352.

INSTITUTE OF MATHEMATICS

POLISH ACADEMY OF SCIENCES

ŁÓDŹ BRANCH

NARUTOWICZA 56

90-136 ŁÓDŹ, POLAND 